\title{
Hypertrophy, heart failure, brain and physical activity - the molecular basis of this connection
}

\begin{abstract}
Physical activity is an important tool to interfere on the natural course of cardiac hypertrophy to heart failure and also to manage this condition once it is established. Aerobic physical activity increases parasympathetic tone while decreases sympathetic activity. This scenario decreases peripheral vascular resistance, alters autonomic nervous system and affect cardiac effort and output. Moreover, exercise increases concentrations of Vascular Endothelial Growth Factor (VEGF) and Brain-derived Neurotrophic Factor (BDNF) on the brain, leading to cerebral vasculature changes, increased neuronal density and enhanced synapses strengthen. In addition, it promotes structural and functional changes on the cardiovascular system and brain. Exercise is widely shown to act on the prefrontal cortex and amygdala functions, which are related to autonomic nervous system, building a feedback system, linking central and peripheral tissues.
\end{abstract}

Keywords: hypertrophy, heart failure, brain and physical activity, molecular, structural and functional changes
Volume II Issue 4 - 2018

\author{
Maria Luíza Medeiros Rêgo, Daniel Aranha \\ Rego Cabral \\ Health Science Center, Federal University of Rio Grande do \\ Norte, Brazil
}

Correspondence: Maria Luíza Medeiros Rêgo, Health Science Center, Federal University of Rio Grande do Norte, Brazil, Email marialuizamedeirosr@gmail.com

Received: July 12, 2018 | Published: July 23, 2018

\section{Introduction}

Heart failure (HF) is a syndrome developed chronically. This disorder represents one of the leading causes of mortality worldwide. ${ }^{1}$ HF condition referred to cardiac muscle impossibility to give cardiac output properly according to body tissues metabolism. Thus, some organs, such as the brain, receive less than necessary to work. The consequence of this situation is a bad functioning or lower cells activity. Talking globally, the patient will suffer of congestion and dyspnea. Thinking more specifically about the brain, the patient might show impaired executive functions. ${ }^{2}$ In sum, HF is manifested as a restriction of body energy, implying in lower activity levels for the patient who will barely execute the daily routine. ${ }^{2}$ Into this context, physical exercise (PE) has been pointed out as an important auxiliary tool in the treatment of patients with HF and previous articles discussed the role played from PE on the brain of HF individuals.

\section{Physiopathology of heart failure}

The HF scenario is established according to neurohumoral mechanisms, ${ }^{3}$ which leads, in a first moment, to a benefic and adaptive situation for the heart pump enough blood to the body. However, looking in a long-term the scenario is an unregulated adaptation. Therefore, we can set 2 points to evaluate the development of heart failure:

a. Renin-Angiotensin system: activation of angiotensin type 1 receptor (AT1) by angiotensin II (Ang II) is a strong stimulus to vasoconstriction, cell growth and secretion of both aldosteron and catecholamines.

When the body is under an Ang II chronic secretion, such as in HF condition, it leads to raised blood pressure, heart muscle cell and fibroblasts hypertrophy, as well as collagen accumulation on interstitial tissues. ${ }^{4}$ This scenario leads to extracellular matrix protein synthesis, ${ }^{5}$ remodeling and replace of functional to scarring tissue within the heart. Furthermore, the increased vasoconstriction causes an ischemia area, thereby provoking more cell death and scar tissue. ${ }^{6,7}$
In addition, AngII is closely linked to inflammatory response. It is expressed and activated by macrophages and cardiac myofibroblasts. ${ }^{4}$ Cardiac myofibroblasts emerge as an answer of cardiac fibroblasts under stress. ${ }^{8}$ They express increased levels of pro-inflammatory and pro-fibrotic factors and, also, raise the enzymes levels, such as matrix metalloproteinases that degrade extracellular matrix and favors fibroblasts migration. ${ }^{4,9}$ It is believed that Ang II activation is involved with the via of growth factor $\beta 1$ (TGF- $\beta 1$ ) and, together, they are involved with cardiac fibroblasts transformation in myofibroblasts and are mediators of collagen deposition, fibrosis and cardiac hypertrophy. ${ }^{1,4}$ Moreover, the heart effect of high levels of aldosteron, which happens in HF pathology, is cardiac muscle hypertrophy, fibrosis, and collagen accumulation. If not interrupted, this process can lead to enlargement of left ventricular cavity and systolic dysfunction. ${ }^{6}$

b. Catecholamines: raised blood catecholamine levels in HF patients is a consequence of higher sympathetic nerve activity. ${ }^{10}$ Catecholamines are neuroendocrine hormones that activate adenylylcyclase to increase cyclic adenosine monophosphate (cAMP) levels. cAMP activates protein kinase A (PKA), which promotes an increase in cytosolic $\mathrm{Ca}^{2+}$ levels and contractile proteins. Taken together, cAMP, PKA and high $\mathrm{Ca}^{2+}$ intracellular content provoke cell growth, fibrosis, arrhythmia and contractile dysfunction. ${ }^{10}$ It is important to know that catecholamine levels are inversely correlated with patient survival, once hyperactive autonomic nervous system (raised catecholamine levels) will be an over stimuli to heart muscle pump under a unbalanced condition. And, the heart is not able to handle too much pressure, suffering remodeling. Then, catecholamine effects become toxic for the heart and markedly increase its morbidity and mortality. ${ }^{3}$

A studied demonstrated that under norepinephrine effects, the heart suffers hypertrophy, especially on the left ventricle. In addition, there is a raised levels of atrial natriuretic peptide, higher expression of collagen I and III and interstitial fibrosis. ${ }^{11}$ Characteristically, catecholamines enhance the stimulus to oxidative 
pentose phosphate pathway, which can be certificated by glucose-6phosphate dehydrogenase levels. ${ }^{11}$ After an interval of 4 to 12 hour of a noradrenalin injection in animal model, it was also observed higher levels of interleukin (IL)- 6 and IL-1 $\beta$, molecules involved on the development of cardiac hypertrophy and fibrosis. ${ }^{11}$

\section{Mechanisms of hypertrophy on heart failure}

The hypertrophy is the initial heart reaction against to progressive stress. Actually, depending of the situation, the remodeling mechanism can occur following a physiological way or a pathological one, being associated to higher risk of decompensation, ventricular dilatation, systolic dysfunction, and electrophysiologic and arrhythmia, ${ }^{12}$ and we will discussed about this last one. Nowadays, it is established that changes in cardiac extracellular matrix and the remodeling process play a major role in the development and evolution of cardiac fibrosis, leading to HF. ${ }^{13,14}$ Biochemical mechanisms and signaling pathways are being studied, but some points still remain unclear. Below we discussed about what is known on the hypertrophic mechanisms:

Trying to supply the body with properly blood flow and avoid the lack of tissue nutrition, the heart muscle adapts itself through a remodeling process by muscle growth, inflammation, and angiogenesis, ${ }^{1}$ looking to be stronger and be able to pump appropriately. Initially, cardiac muscle cells increase contractility. This process occurs through the parallel addition of sarcomere units and raised left ventricular wall thickness. ${ }^{10}$ Taken together, these alterations decrease left ventricular wall stress according to Laplace's law. ${ }^{10}$ The Laplace's law shows that a wall stress (WS) of a sphere is proportional to the intracavity pressure $(\mathrm{P})$ and the radio value of this sphere (R), and inversely proportional to the wall thickness $(\mathrm{T})$, given by the formula $\mathrm{WS}=$ $\mathrm{P} * \mathrm{R} / 2 \mathrm{~T}$. Applying into the heart scenario, the ventricle corresponds to the sphere, in means that the cardiac hypertrophy increases " $T$ " value while decreases " $R$ " value. Hence, at least initially, the heart maintains cardiac efficiency. However, after sustained stress, cardiac remodeling leads to a progressive and irreversible dysfunction of the heart, ${ }^{12}$ raised by interstitial fibrosis and cellular apoptosis. ${ }^{15}$

\section{Molecular and ultrastructural analysis}

The molecular environment of remodeling found on $\mathrm{HF}$ is a mechanical stress in addition to not balanced of pro- and antifibroticfactors, where should be found cytokines and chemokines, growth factors, proteases, hormones, and reactive oxygen species. ${ }^{14}$

Among the molecules that are secreted and regulate the extracellular matrix remodeling process, we can mention:

a. TGF- $\beta 1$ : induces collagen deposition; transform fibroblasts to myofibroblasts; increases extracellular matrix proteins synthesis and reduces their degradation. ${ }^{16}$

b. Renin-Angiotensin system: contributes to hypertrophy and fibrosis; up regulates TGF- $\beta 1$ expression; stimulates fibroblasts proliferation, collagen, fibronectin and laninin expression. ${ }^{16}$

c. Matrix metalloproteinases: degradates extracellular matrix components and modulate collagen synthesis. ${ }^{16}$

d. Tumor necrosis factor- $\alpha$ : induces matrix metalloproteinases gene activation and upregulates its expression. ${ }^{16}$

Beginning with smaller structures, analyzing cardiomyocytes from a failed heart, these cells have a low number of myofibrils, compared with enhanced cytoplasm and nuclei, small-sized mitochondria grouped, and proliferation of $\mathrm{T}$ tubules. ${ }^{17,18}$ A study which use electron microscopy and immunocy to chemistry to analyzed the failed heart showed that the vinculin (a protein closely associated with the cytoskeleton), occurred not only at the sarcolemma and the intercalated disc, its normal place to be founded, but also within the myocardial cells, evidencing cytoskeleton changes, that were found also with molecules of desmin, tubulin, and vimentin. ${ }^{18}$

\section{Extracellular matrix analysis}

Going through a bigger scale, the organization of extracellular matrix, compositions and structure, are crucial to maintain the heart functionality, micro and macroscopically, once it is responsible to keeps molecular signaling and mediates cells communication. ${ }^{19}$ All of these factors cited above (TGF- $\beta 1$, Renin-Angiotensin system, Matrix metalloproteinases and Tumor necrosis factor- $\alpha$ ) lead to extracellular matrix reorganization, especially on collagen turnover and the transformation of fibroblasts in myofibroblasts. Not only fibroblasts are able to suffer differenciation on myofibroblasts, but also circulating macrophages, smooth muscle cells, endothelial and fibrocytes. ${ }^{20-22}$ The myofibroblasts express contractile protein $\alpha$ smooth muscle actin that contributes to pathological remodeling., ${ }^{414}$ Also produce procollagen molecules, that will be convert in collagen fibers on the extracellular matrix. ${ }^{19} \mathrm{~A}$ lot of molecules can be cites as components of collagen production mediator or cross-linked process, such as lysyl-oxide, secreted protein acidic and rich in cysteine, tissue transglutaminase and tenascin C. ${ }^{23,24}$ Taking to higher collagen crosslinked, inducing higher myocardial tensile strength. ${ }^{14}$ Regardless of the etiology, fibrosis leads to myocardial stiffness, and this scenario provokes contractile dysfunction, and HF. ${ }^{15}$

Pathological hypertrophy leads to cardiomyocytes lengthening and ventricular dilatation (eccentrichypertrophy) with impaired contractile function. It often results in heart failure with either preserved or reduced ejection fraction. ${ }^{10}$ Hypertrophy process is raised as a consequence of inflammation and apoptosis, which are basis for fibrosis, both interstitial and perivascular. Analyzing deeply, all the remodeling process leads to lower expression of vascular endothelial growth factor (VEGF). In this way, at the same time that fibrosis happens, the capillary density is not able to follow the same progress, promoting ischemia and infracted myocardium. At this time, we have a heart hemodynamically overloaded. ${ }^{1,16}$ Importantly, the remodeling process occurs not only at a restricted site, but also it is spread around. ${ }^{4}$ There is a mechanism known as reactive fibrosis, ${ }^{25,26}$ which means that myofibroblasts cells activate on the scar tissue keep releasing proinflammatory and prohypertrophic signals, that cause necrosis and fibrosis, remodeling the extracellular matrix., ${ }^{426,27}$

\section{Chronic stage}

On the chronic fibrosis, an important tool is the inflammation. There is a higher monocyte infiltration that increases the macrophage quantity on the local. These cells are responsible to release amount of cytokine that incites and inflammatory response and activate more macrophages and lymphocytes. ${ }^{28}$ Moreover, another strongly contributor to inflammation are endothelial cells, which turn on a proinflammatory secretory phenotype, creating a profibrotic inflammatory environment. ${ }^{29}$ In addition, the endothelial cells participate directly of the myofibroblasts transdifferentiation, as previously mentioned. ${ }^{1}$ 
Lastly, calcium dysregulation, beyond early macroscopic effects of systolic dysfunction and arrhythmias, can interfere with processes such as hypertrophic growth, energy metabolism, mitochondrial function, and cell survival. ${ }^{1,30}$ In a bigger scale, we have that fibrosis impairs contractility and disturbs the chemo electrical conductance of the heart, leading to arrhythmias, local micro fibrillations, and inefficient contraction. ${ }^{1,31}$

\section{Brain and heart failure}

The brain is one of the major organs that suffers from the heart muscle failure. ${ }^{2}$ It is common we find an association between HF and cerebral dysfunction. Approximately $75 \%$ of this population suffer from cognitive impairment, especially on executive functions domain. ${ }^{32}$ For executive functions we can understand the memory task, planning and execute actions, solve problems, processing speed and inhibitory control. ${ }^{33}$ For a daily routine of patients, this disability is considerably harmful, which means less ability to perform daily activities such as shopping, feeding, establishing relationships, as well as increased morbidity and mortality in this pathology. ${ }^{2}$ For instance, a study analyzed $246 \mathrm{HF}$ patients with whom they measured cognitive ability through Montreal Cognitive Assessment and also estimate cardiovascular risk. They made a follow up of 180 days and, at the end of this half year, they established a link between worse cognitive outcome and higher cardiovascular risk into this sample. They also gave a new utility for brain measurements, showing cognitive dysfunction as a predictor of negative cardiovascular outcomes. ${ }^{34}$

\section{Hypoperfusion and white matter changes}

A recent review analyzed different studies of imaging and summarizes some patterns of alterations that happen on the brain of $\mathrm{HF}$ individuals. They noticed that these individuals have higher areas of white matter hyper intensities. These areas are already linked to decline in global cognition and to higher risk for developing depression, anxiety, cerebrovascular events and dementia. ${ }^{35}$ In addition to the hyper intensities, the studies found that more damages on the white matter, such as myelin pallor, as well as loss of myelin and axons (meaning tissue rarefaction) and mild gliosis. Moreover, the sustained white matter hypo perfusion harms the blood-brain-barrier (BBB) and create a possibility for the plasma spread among the white matter. ${ }^{35} \mathrm{It}$ has been discussed that some of the most important factors that lead to brain changes are cerebral hypoperfusion, as a consequence of lower cardiac output and cerebral microemboli development. Comparing imaging exams of HF patients with a control group matched by aged, areas such as hippocampus, occipital and temporal lobes showed atrophy and alterations on neural networks at resting-state. ${ }^{36}$ The hypoxia environment activates the hypoxia inducible factor-1, which migrates from the cytoplasm to the nucleus and activates a bond of genes linked to hypoxia adaptation. These responses include expression of glucose transporter-1, that alters the neuronal metabolism source, erythropoietin, a pro-survival factor, and activates prodeath genes, such as $\mathrm{p} 53 .{ }^{35,37}$ At the end, the hypoxia causes aggression on the tight junctions and break the BBB. ${ }^{35}$ Furthermore, the hypoxia inducible factor- 1 acts above the astrocytes cells, increasing the VEGF production, which enhance the BBB permeability, changing the composition of interstitial and cerebrospinal fluids. ${ }^{37-39}$ As a consequence of fluid composition changed, there is also an alteration on the aquaporin- 4 receptor (aquaporin- 4 receptors are pumps that regulate molecule crossing, regulating the composition of interstitial and cerebral fluid).$^{38}$ These changes activate astrocytes and microglia that, on the last instance, lead to neuroinflammation, edema, oxidative impairments, neuronal dysfunction and raised production of amyloid precursor protein. ${ }^{40}$ Curiously, higher levels of amyloid precursor protein and amyloid beta protein are associated with lower memory and Alzheimer development. ${ }^{41,42}$

\section{Inflammation}

A recent studied added some other possible mechanisms in order to explain the cognitive impairment into this population, once subclinical dysfunction on left ventricle also developing with neuropsychological harms and subtle vascular brain injury. ${ }^{43}$ It points for possible underlying mechanisms that contributes to brain aggression, such as systemic inflammation, oxidative damage, homocysteinemia, elevated B-type natriuretic peptide, neurohormonal dysfunction, altered cellular metabolism, and impaired cerebrovascular reactivity. ${ }^{35}$

Regarding the inflammatory process, cytokines can provoke the central nervous system for indirect or direct ways. An indirect path occurs through stimuli on peripheral vagal afferents that have projections for amygdala, and a directly path is via hippocampus. ${ }^{35}$ Both of them raise the cytokines derived from astrocytes and glia into the nervous system. Coming from peripheral tissues, interleukin- 1 can cross the $\mathrm{BBB}$ and acts on the cognitive process. ${ }^{44}$. Lastly, cytokines decrease VEGF and BDNF production, implying negative effects on the neurogenesis and brain plasticity. ${ }^{45}$

\section{Autonomic nervous system}

Different mechanisms can explain the cognitive dysfunctions in HF patients, such as cerebral hypoperfusion, atrophy and loss of gray matter of the brain, as well as autonomic nervous system (ANS) dysfunctions. ${ }^{46}$ An important pillar of HF physiopathology that has been previously discussed is the ANS over activation. This means that there is a huge sympathetic activity at the same time that parasympathetic tone is decrease on the organism. ${ }^{47}$ As a systemic result, the body is exposed to higher vasoconstriction and it difficulty the blood to arrive diverse body systems, such as the brain, thus implying cognitive deficits. ${ }^{48,49} \mathrm{~A}$ study with image exams has already shown that patients classified as functional class II (which means that they are comfortable at rest and have slight limitation for physical activity, because of fatigue, palpitation, or dyspnea) had lower function in some important brain regions for cognitive process and autonomic control. As example can be cited hippocampus (short-term memory conversion in long-term memory), caudate nucleus (modulation of body movements), PFC (executive functions) and hypothalamus. ${ }^{46}$

\section{Physical exercise}

Physical exercise (PE) has been suggested as an important auxiliary tool for improve general health and for rehabilitation several pathologies. ${ }^{50}$ It has also been pointed as an adjuvant treatment for HF patients. ${ }^{2}$ Previous researches have discussed the role of PE on the brain of HF individuals. The positive effects promoted by PE could be attributed as a consequence of improvements on cardiac muscle contractile activity, in addition to cause lower peripheral vascular resistance (as a result of a mechanism that decreases sympathetic activation). ${ }^{51}$

\section{Autonomic nervous system}

Studies already had discussed that HF pathology is a condition in which is seen a sympathetic hyperactivity. Enhancement of excitatory inputs including changes in peripheral baroreceptor and chemoreceptor reflexes, chemical mediators that control sympathetic outflow and the 
reduction of sympatho inhibitory reflex..$^{52}$ following this line, some studies have shown that $\mathrm{PE}$ has the ability to reduce sympathetic tone, being important for the development of HF pathology. A research team spent more than 10 years studying the link between ANS and $\mathrm{HF}$ and they demonstrated that regularly-performed exercise training could normalize the hyper activation of sympathetic nerve analyzed by microneurography. ${ }^{53}$ Moreover, it has been observed that HF individuals, functional class II and III, by New York Heart Association (NYHA), found that exercise training promotes a dramatic reduction on directly recorded resting sympathetic nerve activity. ${ }^{54}$ These data are important to elucidate how PE is important as part of the management of this chronic syndrome, once lower sympathetic activation might means lower peripheral vascular resistance, which is a strongly harmful topic on the physiopathology of the disease. Lowering vascular resistance can be a way to enhance blood perfusion and avoid ischemia damages.

\section{Neurotrophins and angiogenesis}

As previously said, PE plays a major role on HF rehabilitation, since it modulates some brain regions of fundamental importance in cognitive processes. It is been hypothesized that aerobic physical activity has the ability to increase brain-derived neurotrophic factor $(\mathrm{BDNF})^{55}$ and that is a possible way that PE benefits cognition. This molecule is known to participate on neurogenesis, neuronal survival and synapses strengthening. It has also been proved that BDNF gene and its protein expression are sustained on the hippocampus after exercise even 2 weeks later. ${ }^{56}$ In addition to BDNF, vascular endothelial growth factor (VEGF) has shown to increase as a consequence of PE. As a result of VEGF action, vascular endothelial cells proliferate and migrate, thus raising angiogenesis. ${ }^{56}$ Together these molecules participate on the cognitive improvement process. BDNF acts on neuronal plasticity strengthening of synaptic connections and VEGF raises the angiogenesis process. There are also studies showing the prefrontal cortex as a target of higher perfusion under exercise condition. Interestingly, increased prefrontal cortex activity has been associated with improved ANS control and increased vagal outflow. The frontal lobe region projects its axons to the amygdala, thus promoting an inhibitory function over this sub cortical area. Hence, the inhibited amygdala stops inhibiting parasympathetic neurons and starts to inhibit adrenergic ones, favoring lower peripheral resistance and better blood flow. ${ }^{57}$

\section{Cerebral blood flow}

There is evidence to demonstrate that HF patients have lower cerebral blood flow. For instance, it has been reported that HF patients have this condition when they go from the sitting to upright position. It is discussed that on sitting position, the individuals have poor cardiac output. As a consequence, simple activities, such as change positions can be accompanied of considerable hypo perfusion. ${ }^{49}$ Interestingly, this scenario evidence the lower oxygen supply to the brain and might explain, for example, the cognitive impairment seen in this pathology because of cerebral hypo perfusion. In this context, an interesting review showed that aerobic exercise can improve cardiovascular function and raise cerebral blood flow, which is an important tool to avoid cognitive impairment because increase nutrient and oxygen delivery to the neurons. ${ }^{58}$ To corroborate with the above-mentioned, researchers evaluated the blood oxygen levels of HF patients and verified that in resting-state, the fluctuations of blood oxygen was associated with the oscillations of the arterial blood pressure and cerebral blood flow. ${ }^{58}$ This strengthens the relation between cardiovascular system, heart and cerebral blood flow and corroborates with the hypo perfusion being a cause of cognitive dysfunction. A neuron image study used in animal model observed that those mice that exercised for 2 weeks increased the total blood volume on the brain, especially on dentate gyrus (a hippocampus' area). In addition, the same work showed spread on new blood vessels created because of angiogenesis-induced by PE. ${ }^{59}$

\section{Neurogenesis}

Another point to be raised is the $\mathrm{PE}$ ability to promote neurogenesis. It is well-documented that, in healthy individuals, aerobic exercise promotes the proliferation of new neurons on the hippocampus. For instance, it has been demonstrated that individuals with higher aerobic fitness have greater bilateral hippocampus volume. ${ }^{60}$ In the context of HF, researchers submitted patients with congestive HF, classified as Functional Class III (NYHA) to an exercise training program during 18 weeks, twice a week. In addition, they conducted a cognitive test battery to assess cognitive functions. It was noted, after the intervention, that patients improved general attention and psychomotor speed compared to the baseline. ${ }^{61}$ In order to show different ways that physical activity can improve cognition not only by cerebral blood flow, a study demonstrated that exercise up regulates neuronal proliferation, strengths length, complexity, density of granule cell dendrites and reinforces neuronal plasticity. ${ }^{56}$ One of the studied areas of neurogenesis process as a consequence of exercise was the hippocampus, whereas PE increases neurogenesis, boosting memory and learning capacity. ${ }^{6}$

\section{Peripheral Effects}

It is worth mentioning that $\mathrm{PE}$ also has antihypertensive effects. This phenomena is described as post-exercise hypotension and it is observed both acutely and chronically as a body physiological consequence of exercise. ${ }^{63}$

Acutely, it has been reported reductions of BP in both aerobic and resistance training. ${ }^{64,65}$ Chronically, previous studies found that regularly-performed $\mathrm{PE}$ is associated with reductions of 8.3 and 5.2 $\mathrm{mmHg}$ in resting systolic and diastolic BP, respectively ${ }^{66}$ Corroborating with this data, evidence that summarizes the effects exercise training, showing that the effects of $\mathrm{PE}$ reach a reduction greater than $5 \mathrm{mmHg}$ on systolic blood pressure. ${ }^{67} \mathrm{~A}$ possible explanation for this lower blood pressure effect of exercise is the decrease of systemic vascular resistance.$^{68}$ Considering that the systemic levels of arterial pressure means the after load, which is the force of opposition that the cardiac muscle needs to pump to offer properly blood flow for the body and that it is already established an inverse relation between after load and systolic performance. ${ }^{69}$ Thus, a reduction in this value means a relief or a lower "load" against which the heart ejects blood. Therefore, PE can be useful to avoid the progression of HF, once it reduces one of the factors that overload the heart making it strain each time more.

\section{Conclusion}

The main point of this article was create a link between molecular harms of HF pathology, its implications to the brain and the way that possibility physical exercise improve HF condition and its cognitive outcomes. Here, it were highlighted the biggest paths - autonomic 
nervous system, hemodynamic and molecular patterns - to explain HF and physical exercise effects to the brain.

\section{Acknowledgments}

None.

\section{Conflict of interest}

The author declares that there is no conflict of interest.

\section{References}

1. Schirone L, Forte M, Palmerio S, et al. A Review of the Molecular Mechanisms Underlying the Development and Progression of Cardiac Remodeling. Oxid Med Cell Longev. 2017;3920195.

2. Maria Luíza de Medeiros Rêgo, Daniel Aranha Rego Cabral, Eduardo Bodnariuc Fontes. Cognitive Deficit in Heart Failure and the Benefits of Aerobic Physical Activity. Arq Bras Cardiol. 2018;110(1).

3. Lymperopoulos A, Rengo G, Koch WJ. Adrenergic nervous system in heart failure: pathophysiology and therapy. Circ Res. 2013;113(6):739753 .

4. Travers JG, Kamal FA, Robbins J, et al. Cardiac Fibrosis: The Fibroblast Awakens. Circ Res. 2016;118(6):1021-1040.

5. Villarreal FJ, Kim NN, Ungab GD, et al. Identification of functional angiotensin II receptors on rat cardiac fibroblasts. Circulation. 1993;88(6):2849-2861.

6. KilickıranAvci B, İkitimur B, Karadag B, et al. Renin-angiotensin system blockade in the treatment of heart failure and the role of valsartan in this treatment. Anadolu Kardiyol Derg. 2014;14 (Suppl 2):S1-8.

7. Cohn JN. Role of the renin-angiotensin system in cardiovascular disease. CardiovascDrugsTher. 2010;24(4):341-344.

8. Benjamin EJ, Blaha MJ, Chiuve SE, et al. Heart Disease and Stroke Statistics-2017 Update: A Report From the American Heart Association. Circulation. 2017;135(10):e146-603.

9. Ali SR, Ranjbarvaziri S, Talkhabi M, et al. Developmental heterogeneity of cardiac fibroblasts does not predict pathological proliferation and activation. Circ Res. 2014;115(7):625-635.

10. Nakamura M, Sadoshima J. Mechanisms of physiological and pathological cardiac hypertrophy. Nat Rev Cardiol. 2018;15(7):387-407.

11. Zimmer HG. Catecholamines and Cardiac Remodeling. In: Cardiac Remodeling and Failure. Boston: Springer; 2003. pp.293-304.

12. Hill JA, Olson EN. CardiacPlasticity. N Engl J Med. 2008;358(13):13701380.

13. Weber KT, Sun Y, Bhattacharya SK, et al. Myofibroblast-mediated mechanisms of pathological remodelling of the heart. Nat Rev Cardiol. 2013;10(1):15-26.

14. Gyöngyösi M, Winkler J, Ramos I, et al. Myocardial fibrosis: biomedical research from bench to bedside. Eur J Heart Fail. 2017;19(2):177-191.

15. Jellis C, Martin J, Narula J, et al. Assessment of nonischemic myocardial fibrosis. J Am Coll Cardiol. 2010;56(2):89-97.

16. Segura AM, Frazier OH, Buja LM. Fibrosis and heart failure. Heart Fail Rev. 2014;19(2):173-185.

17. Schaper J, Meiser E, Stämmler G. Ultrastructural morphometric analysis of myocardium from dogs, rats, hamsters, mice, and from human hearts. Circ Res. 1985;56(3):377-391.
18. Schaper J, Froede R, Hein S, et al. Impairment of the myocardial ultrastructure and changes of the cytoskeleton in dilated cardiomyopathy. Circulation. 1991;83(2):504-514.

19. Haas HJ de, Arbustini E, Fuster V, et al. Molecular Imaging of the Cardiac Extracellular Matrix. Circulation Research. 2014;114(5):903-915.

20. Bucala R, Spiegel LA, Chesney J, et al. Circulating fibrocytes define a new leukocyte subpopulation that mediates tissue repair. Mol MedCamb Mass. 1994;1(1):71-81.

21. Zeisberg EM, Tarnavski O, Zeisberg M, et al. Endothelial-tomesenchymal transition contributes to cardiac fibrosis. Nat Med. 2007;13(8):952-961.

22. van den Borne SWM, Diez J, Blankesteijn WM, et al. Myocardial remodeling after infarction: the role of myofibroblasts. Nat Rev Cardiol. 2010;7(1):30-37.

23. Frangogiannis NG. Matricellular proteins in cardiac adaptation and disease. Physiol Rev. 2012;92(2):635-688.

24. Greenberg CS, Birckbichler PJ, Rice RH. Transglutaminases: multifunctional cross-linking enzymes that stabilize tissues. FASEB J. 1991;5(15):3071-3077.

25. Turner NA, Porter KE. Function and fate of myofibroblasts after myocardial infarction. Fibrogenesis Tissue Repair. 2013;6(1):5.

26. Krenning G, Zeisberg EM, Kalluri R. The Origin of Fibroblasts and Mechanism of Cardiac Fibrosis. J CellPhysiol. 2010;225(3):631-637.

27. Vasquez C, Benamer N, Morley GE. The cardiac fibroblast: functional and electrophysiological considerations in healthy and diseased hearts. $J$ CardiovascPharmacol. 2011;57(4):380-388.

28. Frangogiannis NG. Regulation of the inflammatory response in cardiac repair. Circ Res. 2012;110(1):159-173.

29. Fujita S, Shimojo N, Terasaki F, et al. Atrial natriuretic peptide exerts protective action against angiotensin II-induced cardiac remodeling by attenuating inflammation via endothelin-1/endothelin receptor A cascade. Heart Vessels. 2013;28(5):646-657.

30. Burchfield JS, Xie M, Hill JA. Pathological ventricular remodeling: mechanisms: part 1 of 2. Circulation. 2013;128(4):388-400.

31. Prabhu SD, Frangogiannis NG. The Biological Basis for Cardiac Repair After Myocardial Infarction: From Inflammation to Fibrosis. Circ Res. 2016;119(1):91-112.

32. Vogels RLC, Scheltens P, Schroeder-Tanka JM, et al. Cognitive impairment in heart failure: a systematic review of the literature. Eur $J$ Heart Fail. 2007;9(5):440-449.

33. Diamond A. Executive functions. Annu Rev Psychol. 2013;64:135-168.

34. Gelow JM, Mudd JO, Chien CV, et al. Usefulness of cognitive dysfunction in heart failure to predict cardiovascular risk at 180 days. Am J Cardiol. 2015;115(6):778-782.

35. Ampadu J, Morley JE. Heart failure and cognitive dysfunction. Int $J$ Cardiol. 2015;178:12-23.

36. Pressler SJ, Jung M. Chronic Heart Failure With Memory and Attention Dysfunction: Old Problem, Thinking Anew. JACC Heart Fail. 2018;6(7):593-595.

37. Chen W, Jadhav V, Tang J, et al. HIF-1 alpha inhibition ameliorates neonatal brain damage after hypoxic-ischemic injury. ActaNeurochir Suppl. 2008;102:395-399.

38. Iacovetta C, Rudloff E, Kirby R. The role of aquaporin 4 in the brain. Vet ClinPathol. 2012;41(1):32-44. 
39. Reuss B, Dono R, Unsicker K. Functions of fibroblast growth factor (FGF)-2 and FGF-5 in astroglial differentiation and blood-brain barrier permeability: evidence from mouse mutants. $J$ Neurosci. 2003;23(16):6404-6412.

40. Sfera A, Osorio C. Water for thought: is there a role for aquaporin channels in delirium?. Front Psychiatry. 2014;5:57.

41. Hong $\mathrm{X}, \mathrm{Bu} \mathrm{L}$, Wang $\mathrm{Y}$, et al. Increases in the Risk of Cognitive Impairment and Alterations of Cerebral $\beta$-amyloid Metabolism in Mouse Model of Heart Failure. PLoS ONE. 2013;8(5):e63829.

42. Morley JE, Farr SA. The role of amyloid-beta in the regulation of memory. BiochemPharmacol. 2014;88(4):479-485.

43. Hammond CA, Blades NJ, Chaudhry SI, et al. Long-Term Cognitive Decline After Newly Diagnosed Heart Failure: Longitudinal Analysis in the CHS (Cardiovascular Health Study). Circ Heart Fail. 2018;11(3):e004476.

44. Banks WA, Farr SA, La Scola ME, et al. Intravenous human interleukin-1alpha impairs memory processing in mice: dependence on blood-brain barrier transport into posterior division of the septum. $J$ PharmacolExpTher. 2001;299(2):536-541.

45. Yirmiya R, Goshen I. Immune modulation of learning, memory, neural plasticity and neurogenesis. Brain Behav Immun. 2011;25(2):181-213.

46. Woo MA, Kumar R, Macey PM, et al. Brain Injury in Autonomic, Emotional, and Cognitive Regulatory Areas in Patients with Heart Failure. J Card Fail. 2009;15(3):214-223.

47. Nolan J, Batin PD, Andrews R, et al. Prospective study of heart rate variability and mortality in chronic heart failure: results of the United Kingdom heart failure evaluation and assessment of risk trial (UK-heart). Circulation. 1998;98(15):1510-1516.

48. Creager MA, Faxon DP, Cutler SS, et al. Contribution of vasopressin to vasoconstriction in patients with congestive heart failure: comparison with the renin-angiotensin system and the sympathetic nervous system. $J$ Am CollCardiol. 1986;7(4):758-765.

49. Fraser KS, Heckman GA, McKelvie RS, et al. Cerebral hypoperfusion is exaggerated with an upright posture in heart failure: impact of depressed cardiac output. JACC Heart Fail. 2015;3(2):168-175.

50. Sallis RE. Exercise is medicine and physicians need to prescribe it!. $\mathrm{BrJ}$ Sports Med. 2009;43(1):3-4.

51. Galioto R, Fedor AF, Gunstad J. Possible neurocognitive benefits of exercise in persons with heart failure. EurRevAgingPhysAct. 2015;12:6.

52. Kishi T, Hirooka Y. Central Mechanisms of Abnormal Sympathoexcitation in Chronic Heart Failure. CardiologyResearchandPractice. 2012.

53. Antunes-Correa LM, Melo RC, Nobre TS, et al. Impact of gender on benefits of exercise training on sympathetic nerve activity and muscle blood flow in heart failure. Eur J Heart Fail. 2010;12(1):58-65.

54. Roveda F, Middlekauff HR, Rondon MUPB, et al. The effects of exercise training on sympathetic neural activation in advanced heart failure: a randomized controlled trial. J Am CollCardiol. 2003;42(5):854-860.
55. Dinoff A, Herrmann N, Swardfager W, et al. The effect of acute exercise on blood concentrations of brain-derived neurotrophic factor in healthy adults: a meta-analysis. Eur J Neurosci. 2017;46(1):1635-1646.

56. Baek SS. Role of exercise on the brain. J ExercRehabil. 2016;12(5):380 385 .

57. Thayer JF, Lane RD. Claude Bernard and the heart-brain connection: further elaboration of a model of neurovisceral integration. NeurosciBiobehav Rev. 2009;33(2):81-88.

58. Tarumi T, Zhang R. Cerebral Blood Flow in Normal Aging Adults: Cardiovascular Determinants, Clinical Implications, and Aerobic Fitness. J Neurochem. 2017;144(5):595-608.

59. Pereira AC, Huddleston DE, Brickman AM, et al. An in vivo correlate of exercise-induced neurogenesis in the adult dentate gyrus. ProcNatlAcadSci U S A. 2007;104(13):5638-5643.

60. Erickson KI, Prakash RS, Voss MW, et al. Aerobic fitness is associated with hippocampal volume in elderly humans. Hippocampus. 2009;19(10):1030-1039.

61. Tanne D, Freimark D, Poreh A, et al. Cognitive functions in severe congestive heart failure before and after an exercise training program. Int J Cardiol. 2005;103(2):145-149.

62. van Praag H. Neurogenesis and exercise: past and future directions. Neuromolecular Med. 2008;10(2):128-140.

63. Costa EC, Dantas TCB, de Farias Junior LF, et al. Inter- and IntraIndividual Analysis of Post-Exercise Hypotension Following a Single Bout of High-Intensity Interval Exercise and Continuous Exercise: A Pilot Study. Int J Sports Med. 2016;37(13):1038-1043.

64. Casonatto J, Goessler KF, Cornelissen VA, et al. The blood pressurelowering effect of a single bout of resistance exercise: A systematic review and meta-analysis of randomised controlled trials. Eur $J$ PrevCardiol. 2016;23(16):1700-1714.

65. Carpio-Rivera E, Moncada-Jiménez J, Salazar-Rojas W, et al. Acute Effects of Exercise on Blood Pressure: A Meta-Analytic Investigation. Arq Bras Cardiol. 2016;106(5):422-433.

66. Cornelissen VA, Smart NA. Exercise Training for Blood Pressure: A Systematic Review and Meta-analysis. $J$ Am Heart Assoc. 2013;2(1):e004473.

67. Whelton PK, He J, Appel LJ, et al. Primary prevention of hypertension: clinical and public health advisory from The National High Blood Pressure Education Program. JAMA. 2002;288(15):1882-1888.

68. Brito LC, Queiroz ACC, Forjaz CLM. Influence of population and exercise protocol characteristics on hemodynamic determinants of postaerobic exercise hypotension. Braz J MedBiol Res. 2014;47(8):626-636.

69. LaCombe P, Lappin SL. Physiology, Cardiovascular, Cardiac Output, Afterload Reduction.Treasure Island (FL): StatPearls Publishing; 2018. 